\title{
Engineered microdevices for single cell immunological assay
}

\author{
Jonghoon $\mathrm{Choi}^{1{ }^{*}}$ \\ 1Department of Chemical Engineering, Massachusetts Institute of Technology, 77 Massachusetts Ave., Cambridge, MA 02139
}

Subject areas:Nano-bio and Nanomedical science and technology

Author contribution: Choi $\mathrm{JH}$ designed and wrote this manuscript.

${ }^{*}$ Correspondence and requests for material should be addressed to J.H. C. (jonghoon@mit.edu)

Reviewer: Keun Woo Lee, Gyeongsang National University, Republic of Korea. Hyun Joo, Inje Univ. College of Medicine, Republic of Korea.

Editor: Sun Shim Choi, Kangwon National University, Republic of Korea

Received March 22, 2010;

Accepted April 27, 2010;

Published April 29, 2010

Citation: Choi, J. Engineered microdevices for single cell immunological assay. IBC 2010, 2(2):1, 1-8. doi:10.4051/ibc.2010.2.2.0001

Competing interests: All authors declare no financial or personal conflict that could inappropriately bias their experiments or writing.

Copyright: This article is licensed under a Creative Commons Attribution License, which freely allows to download, reuse, reprint, modify, distribute, and/or copy articles as long as a proper citation is given to the original authors and sources.

\section{SYNOPSIS}

Microdevices have been used as effective experimental tools for the rapid and multiplexed analysis of individual cells in single-cell assays. Technological advances for miniaturizing such systems and the optimization of delicate controls in micron-sized space homing cells have motivated many researchers from diverse fields (e.g., cancer research, stem cell research, therapeutic agent development, etc.) to employ microtools in their scientific research. Microtools allow high-throughput, multiplexed analysis of single cells, and they are not limited by the lack of large samples. These characteristics may significantly benefit the study of immune cells, where the number of cells available for testing is usually limited. In this review, I present an overview of several microtools that are currently available for single-cell analyses in two popular formats: microarrays and microfluidic microdevices. Then, I discuss the potential to study human immunology on the single-cell level, and I highlight several recent examples of immunoassays performed with single-cell microdevice assays. Finally, I discuss the outlook for the development of optimized assay platforms to study human immune cells. The development and application of microdevices for studies on single immune cells presents novel opportunities for the qualitative and quantitative characterization of immune cells and may lead to a comprehensive understanding of fundamental aspects of human immunology.

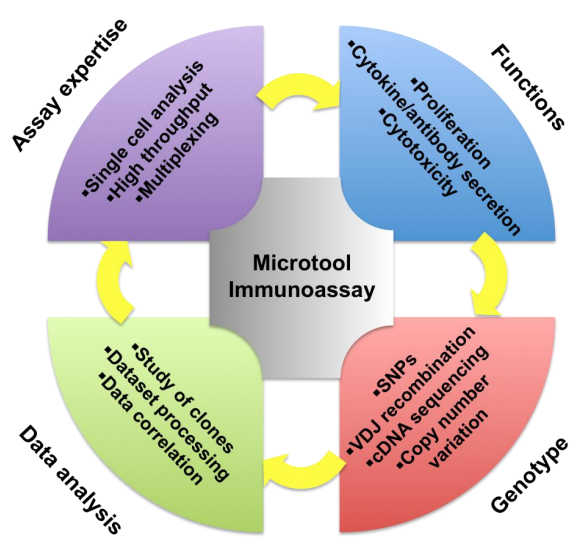

Keywords: single cell assay, microfluidics, microwells, immune response 


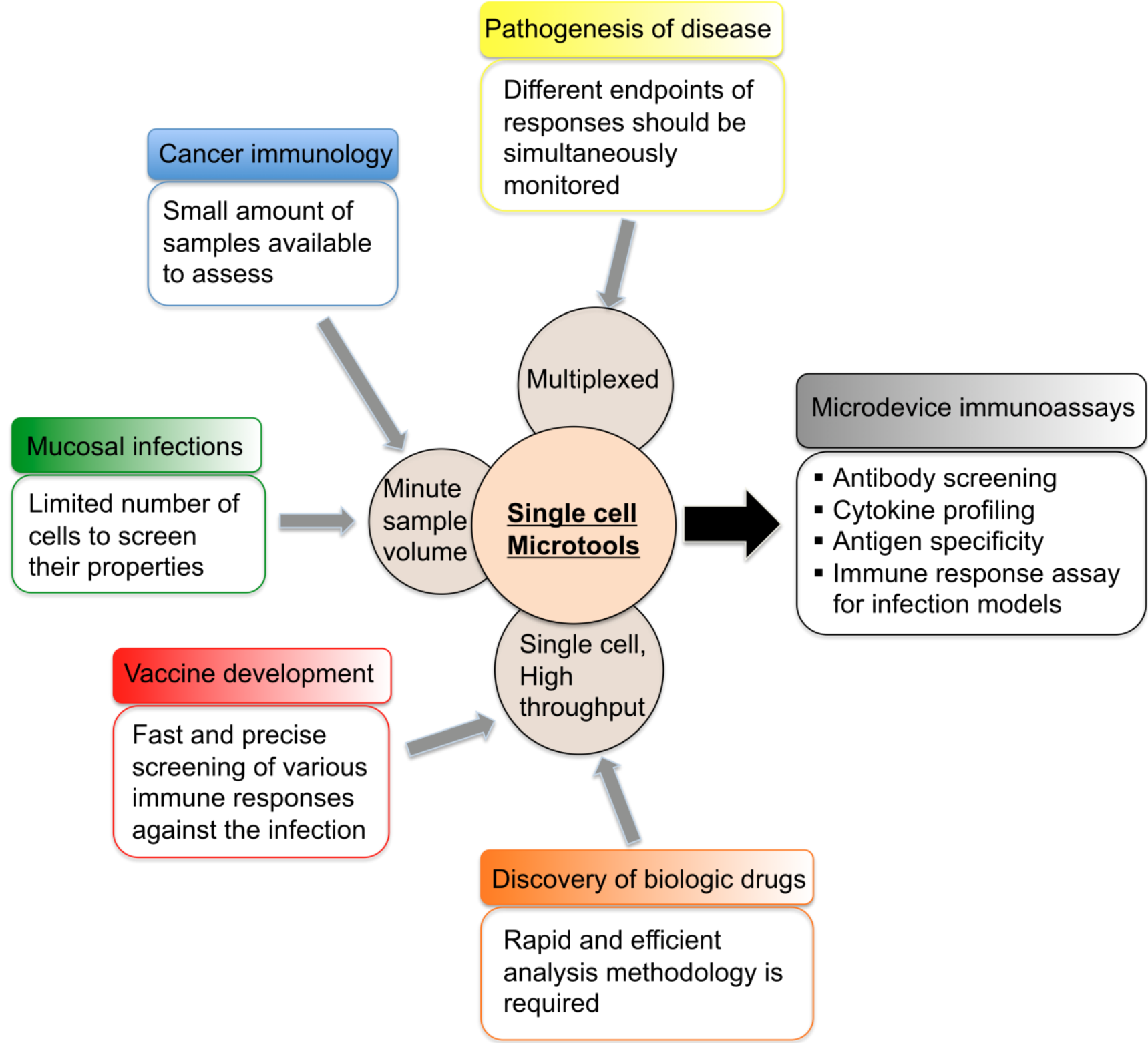

Figure 1. Opportunities of single cell microtools employed in human immunology. Probing immunological diseases and cancer requires an assay that can work with small amount of samples. In order to develop a vaccine and drug, introducing a high throughput and precise assaying method is essential. Since different and multiple proteins can be related to the disease pathogenesis, multiplexed profiling of single immune cells is critically important to understand the infection and immune response mechanism against it. Microdevices (e,g, microwell arrays, microfluidic tools, etc.) for single cell assay are the potential candidate which may answer these requirements for comprehensive investigating of the human immunology and its related problems.

\section{Introduction}

Following initial demonstrations of the utility of microfabrication technology for biological studies, microdevice tools have evolved such that they are now employed in research ranging from basic science to clinical tests (Howard et al., 1983; Voldman et al., 1999). Microdevices provide a platform to study novel experimental designs and provide an appropriate tool for single-cell investigations. Single cells studied with microtools provide information regarding a single cell's life and function; this information, although variable among individual cells, is still generally applicable to all cells of the same type (e.g., proliferation, growth, metabolism, movement, creation of proteins, immunological responses, etc.). Advanced microdevices and tools allow single-cell analysis and are used for various single-cell assays (Bao et al., 2008; Brouzes et al., 2009; Faley et al., 2009; Kumaresan et al., 2008; Love et al., 2006; Mittal et al., 2007; Ochsner et al., 2007; Ogunniyi et al., 2009; Rettig and Folch, 2005; Rowat et al., 2009; Valero et al., 2005; Zhu et al., 2009). In this review, I highlight two major types of microtools: devices based on microwells and microfluidic microsystems. Both types of microdevices share similar characteristics, and range from tens to hundreds of micrometers in size. Microdevices are often fabricated using 'soft-lithography', for which the most commonly used material is polydimethylsiloxane (PDMS) due to its superior elasticity and aerating properties. These qualities make PDMS suitable for biological applications (Bruzewicz et al., 2008; McDonald et al., 2000). Microdevices have been praised for use in rapid analysis due to their high sensitivity, their scalability, and the ease with which many replicate experiments can be performed with minimal reagent and sample consumption. Microdevice platforms also simplify analysis and reduce sample handling, which is useful in biological research in general and is particularly well-suited to single-cell studies. Most existing cell studies are based on bulk- 
scale analysis of a group of cells to achieve statistically meaningful results. However, this approach does not necessarily provide detailed information about the behavior of individual cells. Since microdevices are designed to retain individual cells for a long period of time, microdevices are promising candidates for studying characteristics of single cells that may have been veiled by averaging in bulk. Therefore, microtools may allow study of the characteristics, functions, and genetic information of cells present at low densities (e.g., stem cells) or samples from patients (especially for infant patients). These studies, in turn, may help us to understand and diagnose disease at an early stage. In addition, the development of high-throughput, multiplexed single-cell screening assays to test the immunological response of individual immune cells may assist the design of novel vaccines and expand our understanding of the pathogenesis of various diseases. Specific areas of interest in immunological assays using single-cell microtools will include, but not be limited to, antibody screening, cytokine profiling, antigen specificity tests, and infection model analyses. In this review, I first highlight the opportunities to study human immunology and discuss the limitations of the current methodologies available for these studies when compared to highthroughput, multiplexed single-cell immunoassays. Then, I summarize the two available microdevice platforms, microwell and microfluidic devices, and discuss their characteristics, pros and cons inherent to the nature of the devices, and the requirements and limitations for their application in specific immunoassays. Previous research on immunological assays using these microdevices is summarized and categorized according to the specific endpoints of the assay measurements. Finally, I address the outlook for the development of future microdevices for singlecell immunoassays and the anticipated research projections toward the full understanding of fundamental questions in human immunology and control of immune diseases.

\section{Opprotunities in human immunology}

Human immunology is involved in many aspects of infectious diseases and research related to these diseases. Understanding immunological components and their correlations with various diseases is expected to assist in formulating strategies to cure them. Here I discuss the subunits of human immunology in the context of their characteristics, examples, opportunities and limitations associated with their study on a cellular level. Then, I will explore advantages of using single-cell assay microdevices for the study of human immunology and its related problems (Figure 1).

\section{Cancer immunology}

The first subcategory of human immunology I discuss is cancer immunology. Cancer immunology is, by definition, the study of correlations of immunological responses with the development and progression of cancer. Monitoring and capturing antigens specific to cancer cells is a good example of studies in cancer immunology. Immunological therapies against cancer often depend on an antitumor immune response. Innate immune cells detect a peripheral outburst of malignancies and induce inflammatory signals between immune cells to attract them to cancer cells. Upregulation of released cytokines (e.g., INF-y) from immune cells is an indication that the immune system has been activated. The key immune cells participating in anti-cancer activity are antigen-presenting dendritic cells and stimulated $T$ cells. Therefore, identifying $T$ cells taking part in killing cancer cells and dendritic cells activating those T cells is critically important to the study of the mechanisms associated with immunological cancer therapy. Current methodologies probing the level of cytokines in the patient's blood are often handicapped by their low sensitivity, the inability to handle small sample volumes, and the time-consuming, labor-intensive nature of the assays. Furthermore, characterizing single immune cells to examine their response against the effectors stimulating cell signaling is challenging since most immunology assays are based on measuring cell behavior and interactions on a large scale. New assay methodologies suitable for rapid examination of individual cells and small samples would be quite valuable.

\section{Mucosal infections}

Mucosal membranes are the first vulnerable areas for pathogens to enter the body. The immune system at the surface of mucosal membranes manages immunological responses to defend against infection by signaling which is separate from the host's innate immune system. The activation of the immune system at the mucosal membrane frequently stimulates the body's immune system, which may be a key to understanding the interactions among the mucosal immune defense mechanism, mucosal infections and systemic immune response in the body. Although little research in this area has been reported, it is essential to relate existing antigens to the response of immune cells in mucosal membranes by measuring cytokine production and cell-to-cell signaling. Infection in mucosal membranes induces cell-mediated immune responses by interplay between $\mathrm{CD}^{+}$and $\mathrm{CD}^{+} \mathrm{T}$ lymphocytes. When this defense mechanism is activated, production of immunoglobulin antibodies is increased and provides a defense against pathogens. In order to design vaccines inducing mucosal protection against pathogens in humans, understanding of these signaling mechanisms and monitoring of released cytokines and antibodies are crucial. However, current bulk immunological assay techniques lack the sensitivity, throughput and small-sample handling capability required to effectively study these processes.

\section{Pathogenesis of diseases}

Disease pathogenesis is another area of human immunology that may benefit from a better understanding of the cause and lineage of various infectious diseases in humans (e.g., microbial infection, inflammation, cancer, etc.). Research in disease pathogenesis can be divided into two subcategories based on the origin of the disease: microbe-based diseases, and autoimmune diseases. In order to recognize the pathogens causing diseases, it is important to pinpoint the relevant pathogenic microorganisms and the responsible immune response. How bacterial and viral pathogens thrive in the host body, obtain nutrients, and generate disease are all fundamental questions in the study of pathogenesis. In addition, it is also important to discover a pathogen's means of evading the immunological defense system in the host. New approaches in vaccine developments and therapeutics are usually rooted in the understanding of this pathogenesis. There is no doubt that new tools to study the pathogenesis precisely but efficiently (in terms of time, labor and cost) on a cellular level would greatly aid the design of novel therapeutics and vaccines for treating infectious diseases. Another subunit of human disease pathogenesis is autoimmune disease. Autoimmune disease is caused when the immune system fails to recognize the host's own tissue as "self", but instead views the host's tissue as some sort of foreign matter. Because of this failure in recognition, the host's own cells and tissues are attacked by his or her immune defense system. It is still unclear why this loss of immunological tolerance occurs. The loss of immunological tolerance is often limited to the autoantibodies produced by B cells (Manjarrez-Orduno et al., 2009; Poletaev et al., 2008). Since T cells may not be responsible for the autoimmune response in some cases, this raises additional questions regarding the manner in which different kinds of $\mathrm{T}$ cells behave during autoimmune disease and also during response to normal pathogenic infections. Since cell-specific analysis and monitoring is required for this type of research, it would be highly advantageous to apply new microdevices that can probe individual cell responses and assay immunological responses against effectors originating from the same host. 


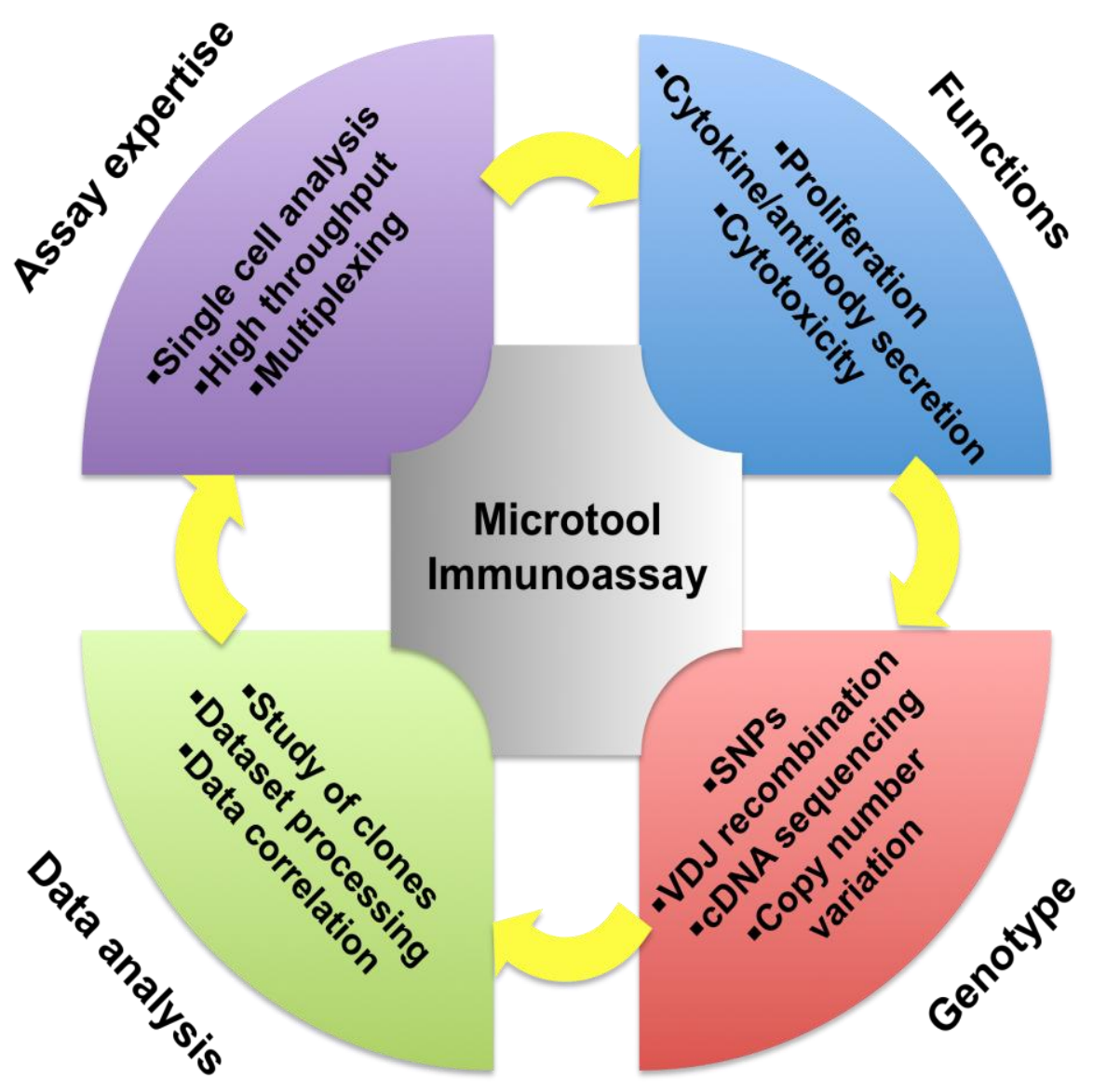

Figure 2. Outlook for the application and practice of microtools in immunoassay. Microtools should embrace three prerequisites as an immunoassay that can analyze individual cells, obtain multiplexed outputs, and perform high throughput screening. Different single cell immunological functions (e.g. proliferation, target molecule secretion, stimulated killing characteristics, etc.) should be probed on chip, simultaneously with a statistical significance. Genotyping of interested single cells and clones should be investigated to illuminate the lineage of functional responses between them. Improvement on data analysis methods should be followed to foster the understanding of complicated networks in immune responses.

\section{Vaccine development}

Research on the pathogenesis of diseases and the characteristics of pathogens has thoroughly examined bacterial and viral infections, but efficient vaccines still do not exist for many infectious diseases (e.g., Helicobacter pylori, HIV, hepatitis C, West Nile virus, etc.). This is mainly because these diseases are difficult to address with current strategies that apply adaptive immunity to identify the viral envelope proteins. New strategies for the design of vaccines should be investigated based on detailed profiling of immune cell responses during their interactions with pathogens. In the case of HIV, the virus can evade the immune defense system by rapidly mutating. The study of this response requires that the immune responses be screened to ensure the most effective and long-term protection from the rapidly mutating virus. In order to develop an effective vaccine, it must first be determined whether specific immune responses against the virus or pathogen can be screened and selected. It will also require researchers to determine whether the combination of two or more antigen-specific antibodies is more effective than use of a single, optimized antibody. Understanding the immune cell signaling mechanism upon exposure to these viruses and pathogens will also be critical to plan a strategy for the development of an effective vaccine. Therefore, the use of microdevices during screening and assessment of immune cells and their signaling mechanisms by might be useful to precisely study the correlations of protection.

\section{Discovery of biologic drugs}

Biological drugs (e.g., antibodies) are different from traditional pharmaceutics (which affect the host's entire immune system) in that they specifically target different portions of the immune system. For example, biological drugs used to treat Psoriatic arthritis are based on TNF-alpha inhibitors that block the production of the protein in the immune system such that the autoimmune response may be reduced. Monoclonal antibodies are good examples of biological drugs that are designed to specifically bind to target substances and inhibit their function. Discovery of these drugs is time-consuming, labor-intensive and costly, and must be improved because future therapeutics may depend heavily on this approach. Rapid screening of antibodies for their specificity using highly sensitive high-throughput analysis of small samples is one area where microdevices may be applied to the biological drug discovery process.

\section{Classes of microtechnologies for single-cell immunoassays}

Microfluidic device 
Microfluidic chips have recently been used in single-cell analysis since they allow precise manipulation of the extracellular environment and satisfy many prerequisites for proper handling of single cells. A variety of cell information can be obtained as cells traverse a microfluidic channel. Cell death, lysis, or morphological variations have been monitored for various types of cells in the microchannel (Nevill et al., 2007). For example, the application of single-cell analysis in a microfluidic channel successfully analyzes a dynamic process of cellular apoptosis (Valero et al., 2005). Microtools have been effective in single-cell analyses where the sample size is usually limited, such as during the study of cell response and viability for primary stem cells (Faley et al., 2009). Microfluidic devices are also a useful system for the study of secretions from single cells. One recent example of such a study is the examination of insulin secretion by murine islet cells encapsulated in droplets (Easley et al., 2009). Technological growth in microfluidic channel design and fabrication has helped researchers to improve their detection of cells by using various modalities. Measurement of electrical signals altered by single cells enabled the quantification and monitoring of cells accurately in microfluidic channels (Bao et al., 2008). Flow cytometric analysis is also possible for single cells passing through narrow microfluidic channels (Huh et al., 2005). PCR can also be performed through the use of microfluidic devices, as shown by efficient detection of fetal chromosomal antibodies from single-cell samples (Fan et al., 2009). As was discussed earlier, the use of microfluidic devices for single-cell analysis is a rapidly growing area of research, and has attracted researchers from many fields of study. Although microfluidic devices can be categorized in many classes as a function of their endpoints and goals, one of the three major types of microfluidic system are often served as an outset. An optimized design and the improvement of various valves and traps required in microfluidic systems have long been discussed in the context of single-cell analysis (Quake and Scherer, 2000; Skelley and Voldman, 2008; Unger et al., 2000). The integration of optical manipulation techniques and other detection systems to the microfluidic chips has further advanced the ability to study single cells with these systems (Diercks et al., 2009; Psaltis et al., 2006). A microfluidic channel is a dynamic system that can simulate a blood vessel or tissue. Development of a continuous perfusion system in the microfluidic channel, therefore, was another major step forward in research of single cells using microtools. A singlecell culture array with the ability to dynamically control fluid was discussed (Di Carlo et al., 2006), and its use for membrane protein analysis and separation of blood cells has been demonstrated on the single-cell level (Fan et al., 2008). A microfluidic device allowing continuous perfusion has also shown its potential for use in cell diagnostics (Myers and Lee, 2008). Droplet-based microfluidic assays are the other type of microfluidic system typically used for analysis of single cells (Brouzes et al., 2009). Trapping single cells in aqueous droplets is advantageous for controlled separation of cells, simultaneous tracking of cell lineages, increasing cell survival percentage, etc. (Rowat et al., 2009). A recent report of a microfluidic fluorescence activated droplet sorter (FADS) described a proof-of-concept experiment that integrated a common cell-sorting method with the advantages of microfluidic cell culture and cell manipulation in microenvironments.

\section{Microarray device}

Microarray technology has been a useful tool to study the characteristics of single-cell microenvironments. One of the main advantages in using microarrays for cell analyses is the ease of automation, which reduces cost and labor, is easily scalable, allows study of single-cell responses in a high-throughput manner, and allows production of robust data sets. One report using a microarray device to probe serum antibody properties in patients with rheumatoid arthritis is a good example showing the prospective utility of these microarray methods for highly sensitive, highly efficient single-cell analysis (Hueber et al., 2005). One critical step for microarray analysis of single cells is development of the ability to specifically load cells in wells. As such, much research has focused on development of a method to provide researchers with optimized loading conditions. For example, different cell loading parameters in microwells have been optimized for adherent and nonadherent cells (Rettig and Folch, 2005). Microwell dimensions and seeding number of cells can be adjusted to promote optimal cell culture. In addition, functionalization of the microwell surface has been suggested as an efficient way to home single cells inside the wells. Surface modification of microwells was demonstrated by coating cationic polymers, which helped to specifically adsorb the protein of interest (Kim et al., 2004). The streptavidin-functionalized surface in microwells can then be used to bind nonadherent B cells, thereby increasing their homing efficiency. Because culturing cells in microwells is different than culturing them in a 2D culture plate and may be more similar to the in vivo environment, there has been an effort to probe cells' behavior in 3D culture and optimize the culture conditions. Therefore, single-cell culture in a 3D array may simulate a microenvironment that cells experience and may direct their responses. A report on engineering a 3D PDMS microwell array for culturing single cells showed its potential to study various cell behaviors and screen them for specific functions (Ochsner et al., 2007). Application of multiplexed detection methods to a microarray device has also been developed, allowing multiplexed measurements and high-throughput analysis of single cells (Seidel and Niessner, 2008). Successful development of optimized cell culture conditions and a controllable microenvironment in microarray devices has facilitated various studies examining singlecell responses, signaling, cell death, etc. For example, T cell and B cell interactions and the mechanisms of signaling between the two types of cells have been studied by locating cells in a microarray (Kim et al., 2006). Rapid, sensitive detection of cell-cell signaling, peptide fragments and pathogens has been performed on the antibody-functionalized surface of microwells. In particular, localizing a number of single mouse embryonic stem cells in specific locations by patterning in the microarray has been shown to modulate cell-cell signaling (Mittal et al., 2007). Development of microwell surface functionalization methods and controlled localization of cells has helped to decipher the complex cell signaling pathways during defense against pathogens (Kim et al., 2006). Recently, a new method called microengraving has allowed multiplexed analysis of secretions from single cells localized in a microwell (Love et al., 2006; Ogunniyi et al., 2009). A large number of single cells were simultaneously assessed $(10,000-80,000$ cells per plate) and analyzed independently for their response to stimulation. This technique has also been suggested as a way to retrieve cells of interest after the measurement for assessment of post-assay responses and cloning.

\section{Examples of single-cell immunoassays enabled by microtools}

\section{Antibody screening}

Microtools have been adapted for immunoassay of single cells. Antibody screening for single cells is one of the endpoints when studying the immune response of cells. Microwell arrays have been suggested as one of the promising microdevices for assay of antibody secreting cells due to their ability to study single cells individually in a multiplexed, high-throughput format (Mills et al., 2001). A. Jin and others have shown facile screening of more than 200,000 individual antigen-specific antibody-secreting cells with a microarray device (Jin et al., 2009). Antibody-secreting cells against hepatitis B virus and influenza viruses were examined individually and retrieved for further clonal expansion. Another good example of 
antibody screening using single cells is a recent report by J.C. Love and others, who used the microengraving method to isolate a specific monoclonal antibody from single cells (Love et al., 2006). One of the outstanding features of this method is its ability to perform multiplexed analysis of antibody-presenting cells (specificity, isotype, and apparent affinity) in a high-throughput manner (Love et al., 2006; Ogunniyi et al., 2009; Ronan et al., 2009; Story et al., 2008). Microfluidic devices have also been employed to study the immunological response of single cells. Although microfluidic devices are promising for single-cell analysis due to their high throughput, high sensitivity, and minute sample volume requirements, application to immunoassays has been limited because conventional flow cytometric assays have shown superior multiplexing capability. However, recent efforts have been made to improve the multiplexing potential of microfluidic tools for immunoassays. A recent review discusses the use of microbeads carrying DNA, RNA, antibodies, or antigens on their surface to resolve the multiplexing issue (Lim and Zhang, 2007). These surface functionalized beads can be introduced and analyzed in microfluidic channels without losing any of the advantages associated with employing microfluidic tools. A.H. Diercks and others report this approach for their assay of single cells secreting antibodies (Diercks et al., 2009). Optically encoded microspheres functionalized with target-specific antibodies were introduced to the microfluidic channel and bound to the desired targets specifically with high efficiency. Since conventional bulk assays (FACS, ELISPOT, etc.) are popular approaches to immunoassays, there has been some effort to adapt bulk assays to the microscale. For example, B.K. Mckenna and others (McKenna et al., 2009) developed a microfluidic system similar to FACS, but retaining the advantages of a microscale fluidic system (e.g., rapid analysis, single cell analysis with high sensitivity, small sample requirements, etc.). Technical advances in this direction are expected to expand the application of microfluidic devices for capture and recognition of antibodies from single cells.

\section{Cytokine profiling}

Profiling of cytokines released from immune cells is another fundamental area of study in immunoassays. Cytokines deliver signals between cells and serve as active immunological modulators. In response to immunological alterations (e.g., infection), the concentration of several cytokines (e.g., IL-6) in the circulation system is amplified up to a thousand times relative to normal conditions. Cytokines can be categorized by their functions, but their concentration near the affecting cells is as critical a factor in determining their characteristics. Secreted cytokines are present in pico- to nano-liter abundance in the system, and microtools may help to detect those minute amounts. The microengraving method has successfully been used to profile secreted cytokines (IFN-8 and IL-6) from single human peripheral blood mononuclear cells (Bradshaw et al., 2008). Secretion of interleukin 2 and interferon- $\mathrm{z}$ cytokines from CD4 and CD8 T cells was also monitored by a microarray of cytokine-specific antibody spots on a glass slide (Zhu et al., 2008). Correlation of captured cytokines on the glass and microwells containing cells showed its potential for individual cell, on chip, multiparametric analysis of cell functions. The same group also looked at different parameters simultaneously for specific $T$ cells. INF- $\mathrm{\gamma}$ release from $\mathrm{T}$ cells arranged in the microwell was captured on the glass slide (Zhu et al., 2009). Mitogenic activation and immunofluorescent staining performed inside the microfluidic chamber revealed that the IFN-x cytokine signal was co-localized with specific T cells. Recently, the cytosolic calcium ion response of single $\mathrm{T}$ cells was investigated as a function of controlled stimulation through interaction with antigen-functionalized microbeads in a miocrochannel (Kirschbaum et al., 2009). A similar idea of calcium ion-related cell signaling and detection was also demonstrated with $\mathrm{B}$ cells based on the behavior of antigen-induced intracellular $\mathrm{Ca}^{2+}$ mobilization (Kinoshita et al., 2009). These studies suggested a rationale for correlating later cell responses (e.g., protein expression) with early monitoring of $\mathrm{Ca} 2+$ signaling in single immune cells. In turn, this may lead to development of tools detecting various diseases and their pathogenesis.

\section{Relevance of microtools for human immunology}

Although traditional ELISA-based, macro-scale analyses are common for immunoassays of cells, a growing amount of research includes assays of single cells in microscale set-ups. As I discussed before, a miniaturization of the assay is beneficial since the required sample volume decreases as the size of the assay is reduced. Microscale systems enable researchers to work with a small quantity of cells, which may limit the use of traditional, bulk-scale immunoassay techniques. The smaller scale of microtools also saves money by decreasing the amount of costly reagents required to run each assay. Furthermore, microscale assays enhance the sensitivity of measurements when compared to the sensitivity that macro-scale assays can achieve. Since a sample volume collected for secreted factors in microassays is small relative to that in ELISAtype immunoassays, it is less challenging to detect a small amount of secreted targets in the sample without potential interference by other molecules. In addition, secreted products from each cell confined in the microscale space can be detected with a high signal-to-noise ratio without loss of products via dilution into bulk solution. Miniaturization of the assay is also favorable when scaling up the experiment. Since a microchip can accommodate several orders of magnitude more experiments on a single chip than ELISA or other macro-scale experiments can, it is able to harvest much more data in a shorter time, thereby ensuring that the results are more precise. This, in turn, may result in better detection of rare events. A micron-sized assay environment also allows precise handling and manipulation of single cells. Since single cells are confined in a well-defined micro-scale space (e.g., microwells, microfluidic channels, etc.), each single cell can be monitored in detail and stimulated individually in a defined manner. The response of each cell in the confined micro-scale space can be accurately traced as well. For instance, one recent report discusses single cells encapsulated in a droplet of PCR mixture, with custom control of the ratio of primer, cell, and PCR mixture by varying the flow conditions in a microfluidic channel (Kumaresan et al., 2008). Automation of the assay procedure is another advantage of employing microtools in immunoassays. Robotic handling of microtiter plates and glass slides reduces labor and the time required for the assay. The accuracy of the measurement can also be supplemented using an automated and standardized assay system. Microtools with these benefits have demonstrated and will continue to demonstrate their potential from proof-of-principle devices to actual applications in human immunology.

\section{Outlook}

Immunoassay microtools allow high-throughput screening of immune cells by minimizing the consumption of reagents and samples. In the near future, I expect to see continued development of well-founded quantitative assays of immune cell function, along with determination of correlation between protection and/or survival against infectious diseases. Regular adaptation of single-cell microtools to human immunology requires development focused on improving the quality of assay expertise, probing multiple functions of the cell, straightforward genotyping, and efficient data analysis (Figure 2). Assay expertise should be mature enough that one can obtain single immune cell-specific, high-throughput and multiplexed data by running a single assay. Multiplexed measurement is likely to unravel different functions of immune cells including cell 
proliferation, secretions, and stimulated cytotoxicity by effector antigens or antigen-presenting cells. Endpoints of the assay should not only measure the phenotypes of the immune cells, but also investigate genotype variations. One of the fundamental questions regarding this will be examination of DNA sequence variation (single nucleotide polymorphism) in immune cells of interest. Detection of genetic recombination (VDJ recombination) in immune cells would also be important, as site-specific gene modification might lead to the production of various $\mathrm{T}$ cell receptors and immunoglobulins in response to antigen stimulations. Other genotyping approaches should also be assessed for use with microtool assays, such as detecting copy number variation or cDNA sequencing to track innate and/or mutated genes by interacting with antigens. This might help in discovering potential associations between genes in the cell and disease. Last but not least, building and sharing a robust data analysis mechanism should be addressed by individual groups or collaborative efforts. Generalized data analysis platforms with open access would expedite assay processing and reduce cost, time and labor. This, in turn, might ultimately allow competent diagnosis of disease and design of drugs and vaccines. Future single-cell microtool assays encompassing all these requirements will prove to be a productive and reliable experimental platform that may guide us to a universal understanding of human immunology for diagnostic and preventive medical applications.

\section{Acknowledgment}

The author acknowledges Prof. J.C. Love(Department of Chemical Engineering, MIT) for helpful discussion.

\section{References}

Bao, N., Wang, J., and Lu, C. (2008). Recent advances in electric analysis of cells in microfluidic systems. Anal Bioanal Chem 391, 933-942.

Bradshaw, E.M., Kent, S.C., Tripuraneni, V., Orban, T., Ploegh, H.L., Hafler, D.A., and Love, J.C. (2008). Concurrent detection of secreted products from human lymphocytes by microengraving: cytokines and antigen-reactive antibodies. Clin Immunol 129, 1018.

Brouzes, E., Medkova, M., Savenelli, N., Marran, D., Twardowski, M., Hutchison, J.B., Rothberg, J.M., Link, D.R., Perrimon, N., and Samuels, M.L. (2009). Droplet microfluidi technology for single-cell high-throughput screening. Proc Natl Acad Sci U S A 106, 14195-14200.

Bruzewicz, D.A., Reches, M., and Whitesides, G.M. (2008). Lowcost printing of poly(dimethylsiloxane) barriers to define microchannels in paper. Anal Chem 80, 3387-3392.

Di Carlo, D., Wu, L.Y., and Lee, L.P. (2006). Dynamic single cell culture array. Lab Chip 6, 1445-1449.

Diercks, A.H., Ozinsky, A., Hansen, C.L., Spotts, J.M., Rodriguez, D.J., and Aderem, A. (2009). A microfluidic device for multiplexed protein detection in nano-liter volumes. Anal Biochem 386, 30-35.

Easley, C.J., Rocheleau, J.V., Head, W.S., and Piston, D.W. (2009). Quantitative measurement of zinc secretion from pancreatic islets with high temporal resolution using droplet-based microfluidics. Anal Chem 81, 9086-9095.

Faley, S.L., Copland, M., Wlodkowic, D., Kolch, W., Seale, K.T., Wikswo, J.P., and Cooper, J.M. (2009). Microfluidic single cell arrays to interrogate signalling dynamics of individual, patientderived hematopoietic stem cells. Lab Chip 9, 2659-2664.
Fan, H.C., Blumenfeld, Y.J., El-Sayed, Y.Y., Chueh, J., and Quake, S.R. (2009). Microfluidic digital PCR enables rapid prenatal diagnosis of fetal aneuploidy. Am J Obstet Gynecol 200, 543.e541-547.

Fan, R., Vermesh, O., Srivastava, A., Yen, B.K., Qin, L., Ahmad, H. Kwong, G.A., Liu, C.C., Gould, J., Hood, L., et al. (2008). Integrated barcode chips for rapid, multiplexed analysis of proteins in microliter quantities of blood. Nat Biotechnol 26, 1373-1378.

Howard, R.E., Liao, P.F., Skocpol, W.J., Jackel, L.D., and Craighead, H.G. (1983). Microfabrication as a Scientific Tool. Science 221, 117-121.

Hueber, W., Kidd, B.A., Tomooka, B.H., Lee, B.J., Bruce, B., Fries, J.F., Sonderstrup, G., Monach, P., Drijfhout, J.W., van Venrooij, W.J., et al. (2005). Antigen microarray profiling of autoantibodies in rheumatoid arthritis. Arthritis Rheum 52, 2645-2655.

Huh, D., Gu, W., Kamotani, Y., Grotberg, J.B., and Takayama, S. (2005). Microfluidics for flow cytometric analysis of cells and particles. Physiol Meas 26, R73-98.

Jin, A., Ozawa, T., Tajiri, K., Obata, T., Kondo, S., Kinoshita, K., Kadowaki, S., Takahashi, K., Sugiyama, T., Kishi, H., et al. (2009). A rapid and efficient single-cell manipulation method for screening antigen-specific antibody-secreting cells from human peripheral blood. Nat Med 15, 1088-1092.

Kim, H., Cohen, R.E., Hammond, P.T., and Irvine, D.J. (2006). Live lymphocyte arrays for biosensing. Adv Funct Mater 16, 13131323.

Kim, H., Doh, J., Irvine, D.J., Cohen, R.E., and Hammond, P.T. (2004). Large area two-dimensional B cell arrays for sensing and cell-sorting applications. Biomacromolecules 5, 822-827.

Kinoshita, K., Ozawa, T., Tajiri, K., Kadowaki, S., Kishi, H., and Muraguchi, A. (2009). Identification of antigen-specific B cells by concurrent monitoring of intracellular $\mathrm{Ca} 2+$ mobilization and antigen binding with microwell array chip system equipped with a CCD imager. Cytometry A 75, 682-687.

Kirschbaum, M., Jaeger, M.S., and Duschl, C. (2009). Correlating short-term $\mathrm{Ca}(2+)$ responses with long-term protein expression after activation of single T cells. Lab Chip 9, 3517-3525.

Kumaresan, P., Yang, C.J., Cronier, S.A., Blazej, R.G., and Mathies, R.A. (2008). High-throughput single copy DNA amplification and cell analysis in engineered nanoliter droplets. Anal Chem 80, 3522-3529.

Lim, C.T., and Zhang, Y. (2007). Bead-based microfluidic immunoassays: the next generation. Biosens Bioelectron 22, 1197-1204.

Love, J.C., Ronan, J.L., Grotenbreg, G.M., van der Veen, A.G., and Ploegh, H.L. (2006). A microengraving method for rapid selection of single cells producing antigen-specific antibodies. Nat Biotechnol 24, 703-707.

Manjarrez-Orduno, N., Quach, T.D., and Sanz, I. (2009). B cells and immunological tolerance. J Invest Dermatol 129, 278-288.

McDonald, J.C., Duffy, D.C., Anderson, J.R., Chiu, D.T., Wu, H., Schueller, O.J., and Whitesides, G.M. (2000). Fabrication of microfluidic systems in poly(dimethylsiloxane). Electrophoresis 21, 27-40.

McKenna, B.K., Selim, A.A., Richard Bringhurst, F., and Ehrlich, D.J. (2009). 384-channel parallel microfluidic cytometer for rare-cell screening. Lab Chip 9, 305-310.

Mills, J.C., Roth, K.A., Cagan, R.L., and Gordon, J.I. (2001). DNA microarrays and beyond: completing the journey from tissue to cell. Nat Cell Biol 3, E175-178.

Mittal, N., Flavin, S., and Voldman, J. (2007). Patterning of 
embryonic stem cells using the bio flip chip. J Vis Exp, 318.

Myers, F.B., and Lee, L.P. (2008). Innovations in optical microfluidic technologies for point-of-care diagnostics. Lab Chip 8, 20152031.

Nevill, J.T., Cooper, R., Dueck, M., Breslauer, D.N., and Lee, L.P. (2007). Integrated microfluidic cell culture and lysis on a chip. Lab Chip 7, 1689-1695.

Ochsner, M., Dusseiller, M.R., Grandin, H.M., Luna-Morris, S., Textor, M., Vogel, V., and Smith, M.L. (2007). Micro-well arrays for 3D shape control and high resolution analysis of single cells. Lab Chip 7, 1074-1077.

Ogunniyi, A.O., Story, C.M., Papa, E., Guillen, E., and Love, J.C. (2009). Screening individual hybridomas by microengraving to discover monoclonal antibodies. Nat Protoc 4, 767-782.

Poletaev, A.B., Stepanyuk, V.L., and Gershwin, M.E. (2008). Integrating immunity: the immunculus and self-reactivity. $J$ Autoimmun 30, 68-73.

Psaltis, D., Quake, S.R., and Yang, C. (2006). Developing optofluidic technology through the fusion of microfluidics and optics. Nature 442, 381-386.

Quake, S.R., and Scherer, A. (2000). From micro- to nanofabrication with soft materials. Science 290, 1536-1540.

Rettig, J.R., and Folch, A. (2005). Large-scale single-cell trapping and imaging using microwell arrays. Anal Chem 77, 5628-5634.

Ronan, J.L., Story, C.M., Papa, E., and Love, J.C. (2009). Optimization of the surfaces used to capture antibodies from single hybridomas reduces the time required for microengraving. $\mathrm{J}$ Immunol Methods 340, 164-169.
Rowat, A.C., Bird, J.C., Agresti, J.J., Rando, O.J., and Weitz, D.A. (2009). Tracking lineages of single cells in lines using a microfluidic device. Proc Natl Acad Sci U S A 106, 18149-18154.

Seidel, M., and Niessner, R. (2008). Automated analytical microarrays: a critical review. Anal Bioanal Chem 391, 15211544.

Skelley, A.M., and Voldman, J. (2008). An active bubble trap and debubbler for microfluidic systems. Lab Chip 8, 1733-1737.

Story, C.M., Papa, E., Hu, C.C., Ronan, J.L., Herlihy, K., Ploegh, H.L., and Love, J.C. (2008). Profiling antibody responses by multiparametric analysis of primary B cells. Proc Natl Acad Sci U S A 105, 17902-17907.

Unger, M.A., Chou, H.P., Thorsen, T., Scherer, A., and Quake, S.R. (2000). Monolithic microfabricated valves and pumps by multilayer soft lithography. Science 288, 113-116.

Valero, A., Merino, F., Wolbers, F., Luttge, R., Vermes, I., Andersson, H., and van den Berg, A. (2005). Apoptotic cell death dynamics of HL60 cells studied using a microfluidic cell trap device. Lab Chip 5, 49-55.

Voldman, J., Gray, M.L., and Schmidt, M.A. (1999). Microfabrication in biology and medicine. Annu Rev Biomed Eng 1, 401-425.

Zhu, H., Stybayeva, G., Macal, M., Ramanculov, E., George, M.D., Dandekar, S., and Revzin, A. (2008). A microdevice for multiplexed detection of T-cell-secreted cytokines. Lab Chip 8, 2197-2205.

Zhu, H., Stybayeva, G., Silangcruz, J., Yan, J., Ramanculov, E., Dandekar, S., George, M.D., and Revzin, A. (2009). Detecting cytokine release from single T-cells. Anal Chem 81, 8150-8156. 